\title{
Regulation of flagellar, motility and chemotaxis genes in Rhizobium leguminosarum by the VisN/ R-Rem cascade
}

Correspondence

Michael F. Hynes

hynes@ucalgary.ca

Received 9 October 2009

Revised 17 February 2010

Accepted 1 March 2010

\author{
Dinah D. Tambalo, Kate L. Del Bel, Denise E. Bustard, \\ Paige R. Greenwood, Audrey E. Steedman and Michael F. Hynes
}

University of Calgary, Department of Biological Sciences, 2500 University Drive NW, Calgary, AB T2N 1N4, Canada

\begin{abstract}
In this paper, we describe the regulatory roles of VisN, VisR and Rem in the expression of flagellar, motility and chemotaxis genes in Rhizobium leguminosarum biovar viciae strains VF39SM and 3841. Individual mutations in the genes encoding these proteins resulted in a loss of motility and an absence of flagella, indicating that these regulatory genes are essential for flagellar synthesis and function. Transcriptional experiments involving gus $A$-gene fusions in wild-type and mutant backgrounds were performed to identify the genes under VisN/R and Rem regulation. Results showed that the chemotaxis and motility genes of $R$. leguminosarum could be separated into two groups: one group under VisN/R-Rem regulation and another group that is independent of this regulation. Vis $\mathrm{N}$ and $\mathrm{Vis} \mathrm{R}$ regulate the expression of rem, while Rem positively regulates the expression of $f l a A, f l a B, f l a C, f l a D, \operatorname{mot} A, \operatorname{mot} B, \operatorname{che} 1$ and $m c p D$. All of these genes except $m c p D$ are located within the main motility and chemotaxis gene cluster of $R$. leguminosarum. Other chemotaxis and motility genes, which are found outside of the main motility gene cluster (che2 operon, flaH for VF39SM, and flaG) or are plasmid-borne (flaE and $m c p C$ ), are not part of the VisN/R-Rem regulatory cascade. In addition, all genes exhibited the same regulation pattern in 3841 and in VF39SM, except flaE and flaH. flaE is not regulated by VisN/R-Rem in 3841 but it is repressed by Rem in VF39SM. flaH is under VisN/R-Rem regulation in 3841, but not in VF39SM. A kinetics experiment demonstrated that a subset of the flagellar genes is continuously expressed in all growth phases, indicating the importance of continuous motility for $R$. leguminosarum under free-living conditions. On the other hand, motility is repressed under symbiotic conditions.

Nodulation experiments showed that the transcriptional activators Vis $\mathrm{N}$ and Rem are dramatically downregulated in the nodules, suggesting that the symbiotic downregulation of motility-related genes could be mediated by repressing the expression of VisN/R and Rem.
\end{abstract}

\section{INTRODUCTION}

Rhizobia are motile members of the $\alpha$-proteobacteria that are known for establishing nitrogen-fixing symbioses inside the nodules of leguminous plants. These nodule bacteria exist either as free-living organisms in the soil or in association with their host plant. In the soil environment, there is a limited availability of utilizable carbon sources for rhizobia. Most of the organic matter in the soil is resistant to microbial degradation and can also form complexes with other compounds (Morita, 1988). There is a narrow region in the soil, the rhizosphere, that is rich in organic and

Abbreviations: MU, Miller units; TEM, transmission electron microscopy. Two supplementary tables, showing primers used to amplify vis $N$, vis $R$ and rem, and details of fragments used for gene-gus $A$ fusions, and a supplementary figure, showing restriction maps of vis $N$, visR and rem, are available with the online version of this paper. inorganic substances secreted by plants (Walker et al., 2003). The ability of rhizobia to reach the rhizosphere and to colonize plant roots enables these soil bacteria to escape nutrient starvation in the soil (Thorne \& Williams, 1997). The ability to sense nutrients and move towards them would therefore provide a competitive advantage for motile and chemotactic rhizobia over non-motile/non-chemotactic strains. The importance of motility and chemotaxis in the ecology of rhizobia is supported by a number of studies that have shown that non-motile or non-chemotactic strains are less competitive and less efficient in nodulation (Ames \& Bergman, 1981; Soby \& Bergman, 1983; Mellor et al., 1987; Caetano-Anollés et al., 1988; Bauer \& Caetano-Anollés, 1990; Liu et al., 1989; Malek, 1992; Yost et al., 1998).

The motility system of rhizobia differs from the known enterobacterial system in terms of genetic organization, flagellar filament assembly, and mode of flagellar rotation. 
The flagellar regulon, which consists of the flagella, motility, chemotaxis and regulatory genes, of Salmonella enterica and Escherichia coli is located in three and four separate clusters, respectively (Kutsukake et al., 1990; Macnab, 1992). On the other hand, the majority of rhizobial genes involved in flagellar assembly and function are located in a single region in the chromosome of rhizobia (Sourjik et al., 1998; Deakin et al., 1999). In Sinorhizobium meliloti, 51 chemotaxis (che), flagellar $(f l a, f l g, f l h, f l i)$, motility $(m o t)$ and regulatory ( $v i s N$, $v i s R, r e m)$ genes are arranged in a single $56 \mathrm{~kb}$ region in the chromosome (Sourjik et al., 1998; Eggenhofer et al., 2006; Rotter et al., 2006). The flagellar filament of enteric bacteria consists of one flagellin subunit, while the flagellar filaments of S. meliloti and Agrobacterium tumefaciens are assembled from four closely related flagellin subunits (Pleier \& Schmitt, 1989, 1991; Sourjik et al., 1998; Deakin et al., 1999). Rhizobia also differ from enteric bacteria in terms of flagellar movement. The rhizobial flagellum rotates in one direction only, i.e. clockwise, as opposed to the bidirectional rotation of the enteric flagellum (Sourjik \& Schmitt, 1996; Berg, 2003; Sourjik et al., 1998). It has been suggested that the direction of movement is reversed by changes in flagellar rotational speed (Sourjik \& Schmitt, 1996; Miller et al., 2007). This type of flagellar movement is thought to be an adaptation of the rhizobia to the specific condition of swimming in viscous fluids that is prevalent in the soil (Scharf et al., 2001).

While it is advantageous for bacteria to be able to move towards favourable environments and away from detrimental conditions, motility is energetically costly. The bacterial cell expends $2 \%$ of its biosynthetic energy for the synthesis and function of the flagellum. Thus, flagellar gene expression is highly regulated by environmental conditions (Soutourina \& Bertin, 2003). The regulation of genes involved in flagellar assembly and function is well studied in enteric bacteria (Silverman \& Simon, 1974; Shi et al., 1992; Pruss \& Matsumura, 1997; Soutourina et al., 2001; Berg, 2003). On the other hand, the regulation of motilityrelated genes is not well understood in rhizobia. To date, the only member of the rhizobia for which the regulation of the flagellar genes has been analysed is $S$. meliloti (Sourjik et al., 2000; Rotter et al., 2006). The motilityrelated genes of $S$. meliloti are tightly regulated in a hierarchical manner. Class IA includes the LuxR-type master regulatory proteins VisN and VisR, which may act as a heterodimer exerting control over the transcription of rem (class IB) (Rotter et al., 2006; Sourjik et al., 2000). Rem is an OmpR-like transcriptional activator that controls the expression of class II genes, which comprise flagellar assembly and motility genes (Rotter et al., 2006). The class IIA genes fliM and orf 38 have been suggested to regulate the expression of the class III genes, which include flagellin and chemotaxis genes. The class IIB genes $\operatorname{mot} A, \operatorname{mot} B$ and $m o t C$ do not exert transcriptional control over the class III genes (Sourjik et al., 2000).

In this paper, we will describe the roles of VisN/R and Rem in the regulation of motility and chemotaxis genes in Rhizobium leguminosarum under free-living and symbiotic conditions. We performed the studies using strains 3841 and VF39SM because the two strains exhibit substantial differences in pattern of flagellation and swarming motility (Tambalo et al., 2010). Using transcriptional fusions to a large number of genes involved in chemotaxis and motility, we demonstrate that only a subset of these genes is regulated by the VisN/R cascade. We also looked at the kinetics of flagellin and motility gene expression to determine their expression patterns at different growth phases. The experiments revealed that the flagellin genes are continuously expressed, indicating that motility may be continuously needed in $R$. leguminosarum under free-living conditions, though it is downregulated during symbiosis.

\section{METHODS}

Bacterial strains, plasmids and growth conditions. The bacterial strains and plasmids used in this study are shown in Table 1. $R$. leguminosarum strains were cultured in TY medium (Beringer, 1974), while E. coli strains were grown in LB medium (Sambrook et al., 1989). Antibiotic concentrations used to culture R. leguminosarum were: $500 \mu \mathrm{g}$ streptomycin $\mathrm{ml}^{-1}, 30 \mu \mathrm{g}$ gentamicin $\mathrm{ml}^{-1}, 100 \mu \mathrm{g}$ neomycin $\mathrm{ml}^{-1}, 100 \mu \mathrm{g}$ spectinomycin $\mathrm{ml}^{-1}$ and $5 \mu \mathrm{g}$ tetracycline $\mathrm{ml}^{-1}$. E. coli strains were grown in the following antibiotic concentrations: $100 \mu \mathrm{g}$ ampicillin $\mathrm{ml}^{-1}, 50 \mu \mathrm{g}$ kanamycin $\mathrm{ml}^{-1}$, $15 \mu \mathrm{g}$ gentamicin $\mathrm{ml}^{-1}$ and $10 \mu \mathrm{g}$ tetracycline $\mathrm{ml}^{-1}$.

Motility tests. Strains were grown in TY broth for $24 \mathrm{~h}$. Swimming motility was determined using a soft agar motility assay, which was done by inoculating the strains into a motility medium containing $0.3 \%$ agar, $0.01 \%$ yeast extract and $1 \mathrm{mM} \mathrm{MgSO}_{4}$ (Ames et al., 1980; Yost et al., 1998). The swimming motility of each of the mutant strains was compared with that of the wild-type 3-4 days after inoculation. Motility was also confirmed by observation under a phase-contrast microscope.

Recombinant DNA techniques. DNA manipulations were done using standard methods (Sambrook et al., 1989). Restriction endonucleases used in this study were purchased from Invitrogen and used according to the manufacturer's specifications. DNA fragments were isolated from the gel using a QIAquick Gel Extraction kit (Qiagen). Plasmids were isolated from E. coli strains using a GeneJET Plasmid Miniprep kit (Fermentas Life Sciences). Total DNA was isolated from R. leguminosarum strains using an AquaPure Genomic DNA Isolation kit (Bio-Rad Laboratories). Primers were designed by Sigma-Genosys (Sigma-Aldrich) and amplification was carried out using a MultiGene II thermal cycler (Labnet International). Southern blots were performed using a non-radioactive technique with reagents and protocols supplied by Roche Applied Science.

Cloning of visNR and rem genes. The regulatory genes $v i s N$, visR and rem were PCR-amplified from R. leguminosarum and then cloned individually into the vector pCR2.1-TOPO using the TOPO Cloning kit (Invitrogen). The primers used for amplifying these genes are shown in Supplementary Table S1.

Insertional mutagenesis. The three regulatory genes vis $N$, visR and rem were excised from the TOPO vector and then ligated into the suicide vector pJQ200SK (Quandt \& Hynes, 1993). Mutants were constructed by introducing either a spectinomycin-resistance or a kanamycin-resistance cassette (Prentki \& Krisch, 1984; Fellay et al., 1987) (Supplementary Fig. S1) within the regulatory gene. The mutated gene was introduced into the genome of $R$. leguminosarum by homologous recombination, as described by Quandt \& Hynes 
Table 1. Bacterial strains and plasmids used in this study

Abbreviations: $\mathrm{Ap}^{\mathrm{r}}$, ampicillin resistance; $\mathrm{Gm}^{\mathrm{r}}$, gentamicin resistance; $\mathrm{Nm}^{\mathrm{r}}$, neomycin resistance; $\mathrm{Sm}^{\mathrm{r}}$, streptomycin resistance; Sp${ }^{\mathrm{r}}$, spectinomycin resistance; $\mathrm{Tc}^{\mathrm{r}}$, tetracycline resistance.

\begin{tabular}{|c|c|c|}
\hline Strain or plasmid & Relevant characteristics & Source or reference \\
\hline \multicolumn{3}{|l|}{ E. coli strains } \\
\hline $\mathrm{DH} 5 \alpha$ & $\begin{array}{l}\text { EndA1, hsdR17, supE44, thi-1, recA1, gyrA96, relA1 (argF-lacZYA), U169, } \varphi \text { 80dlacZ } \\
\Delta \mathrm{M} 15\end{array}$ & Invitrogen \\
\hline S17.1 & $\mathrm{Sp}^{\mathrm{r}}$. RP4 tra region, mobilizer strain & Simon et al. (1983) \\
\hline \multicolumn{3}{|l|}{ R. leguminosarum strains } \\
\hline 3841 & Biovar viciae, JB300 derivative, $\mathrm{Sm}^{\mathrm{r}}$ & Poole et al. (1994) \\
\hline VF39SM flaA ${ }^{-}$ & flaA:: gusA fusion integrated into the genome of VF39SM, $\mathrm{Sm}^{\mathrm{r}}, \mathrm{Gm}^{\mathrm{r}}, \mathrm{Nm}^{\mathrm{r}}$ & This work \\
\hline $3841 \mathrm{flaA}^{-}$ & flaA:: gusA fusion integrated into the genome of $3841, \mathrm{Sm}^{\mathrm{r}}, \mathrm{Gm}^{\mathrm{r}}, \mathrm{Nm}^{\mathrm{r}}$ & This work \\
\hline $3841 v i s N^{-}$ & $\mathrm{Sp}^{\mathrm{r}}$ inserted into $v i s N$ of $3841, \mathrm{Sm}^{\mathrm{r}}, \mathrm{Sp}^{\mathrm{r}}$ & This work \\
\hline $3841 v^{v i s R^{-}}$ & $\mathrm{Km}^{\mathrm{r}}$ inserted into visR of $3841, \mathrm{Sm}^{\mathrm{r}}, \mathrm{Nm}^{\mathrm{r}}$ & This work \\
\hline $3841 \mathrm{rem}^{-}$ & $\mathrm{Km}^{\mathrm{r}}$ inserted into rem of $3841, \mathrm{Sm}^{\mathrm{r}}, \mathrm{Nm}^{\mathrm{r}}$ & This work \\
\hline VF39SM visN & $\mathrm{Sp}^{\mathrm{r}}$ inserted into visN of VF39SM, $\mathrm{Sm}^{\mathrm{r}}, \mathrm{Sp}^{\mathrm{r}}$ & This work \\
\hline pFAJ1701 & RK2-derived cloning vector carrying $g u s A, \mathrm{Ap}^{\mathrm{r}} \mathrm{Tc}^{\mathrm{r}}$ & Dombrecht et al. (2001) \\
\hline pFus1P & pFus1, par cassette inserted from pRR71-H as a HindIII fragment, $\mathrm{Tc}^{\mathrm{r}}$ & Yost et al. (2004) \\
\hline pRK7813 & Broad-host-range cloning vector, $\mathrm{Tc}^{\mathrm{r}}$ & Jones \& Gutterson (1987) \\
\hline pCRS530 & Contains a promoterless gusA-Nm cassette, $\mathrm{Nm}^{\mathrm{r}}$ & Reeve et al. (1999) \\
\hline pRR71-H & Plasmid carrying RK2 par locus as a HindIII fragment & Easter et al. (1997) \\
\hline pFche1 & EcoRI fragment containing the promoter region of the che 1 operon cloned into pFUS1 & Miller et al. (2007) \\
\hline pFche2 & EcoRI fragment containing the promoter region of the che 2 operon cloned into pFUS1 & Miller et al. (2007) \\
\hline pFus1:: flaA & flaA promoter introduced into $\mathrm{pFus1}, \mathrm{Tc}^{\mathrm{r}}$ & This work \\
\hline pFAJ1701:: flaB & flaB promoter introduced into pFAJ1701, $\mathrm{Ap}^{\mathrm{r}} \mathrm{Tc}^{\mathrm{r}}$ & This work \\
\hline pFus1:: flaC (3841) & flaC promoter from 3841 introduced into pFus1, $\mathrm{Tc}^{\mathrm{r}}$ & This work \\
\hline pFus1:: flaC (VF39SM) & flaC promoter from VF39SM introduced into pFus1, $\mathrm{Tc}^{\mathrm{r}}$ & This work \\
\hline pFus $1:$ motB & $m o t B$ promoter from 3841 introduced into $\mathrm{pFus1}, \mathrm{Tc}^{\mathrm{r}}$ & This work \\
\hline pFAJ1701:: motB & $m o t B$ promoter introduced into pFAJ1701, $\mathrm{Ap}^{\mathrm{r}} \mathrm{Tc}^{\mathrm{r}}$ & This work \\
\hline pFus1:: mcpC & $m c p C$ promoter introduced into $\mathrm{pFus} 1, \mathrm{Tc}^{\mathrm{r}}$ & Yost et al. (2004) \\
\hline pFus1:: mcpD & $m c p D$ promoter introduced into pFus1, $\mathrm{Tc}^{\mathrm{r}}$ & Yost et al. (2004) \\
\hline pFus $1:$ :rem $(3841)$ & rem promoter from 3841 introduced into pFus1, $\mathrm{Tc}^{\mathrm{r}}$ & This work \\
\hline pFus1::rem (VF39SM) & rem promoter from VF39SM introduced into pFus1, $\mathrm{Tc}^{\mathrm{r}}$ & This work \\
\hline pFus1:: visN (3841) & visN promoter from 3841 introduced into pFus1, $\mathrm{Tc}^{\mathrm{r}}$ & This work \\
\hline pFAJ1701 : : visN (3841) & visN promoter from 3841 introduced into pFAJ1701, $\mathrm{Ap}^{\mathrm{r}} \mathrm{Tc}^{\mathrm{r}}$ & This work \\
\hline pFus1 : : visN (VF39SM) & $v i s N$ promoter from VF39SM introduced into pFus1, $\mathrm{Tc}^{\mathrm{r}}$ & This work \\
\hline
\end{tabular}

(1993). Southern hybridization was done for each gene to confirm replacement of the wild-type gene with the mutated gene.

Transmission electron microscopy (TEM). Electron microscopy was performed by slightly modifying the procedure of Miller et al. (2007). The regulatory mutants were grown on TY plates at $30^{\circ} \mathrm{C}$ for $48 \mathrm{~h}$. A culture suspension was prepared using sterile double-distilled water. A Formvar-carbon-coated grid was placed on top of a cell suspension drop for $3 \mathrm{~min}$ and excess liquid was removed. Staining was performed using $1 \%$ uranyl acetate for $30 \mathrm{~s}$. Samples were observed using a Hitachi 7650 transmission electron microscope and images were taken using the AMT Image Capture Engine.

Construction of gene fusions and $\beta$-glucuronidase (gus $A$ ) reporter gene assays. To construct gene fusions, the promoters of each of the genes were cloned upstream of a promoterless gusA gene in the vector pFus1 (Reeve et al., 1999), as described by Yost et al. (2004). The regulatory genes vis $N$ and visR are cotranscribed in 
one operon in S. meliloti (Sourjik et al., 2000), and their proximity to each other in R. leguminosarum (Young et al., 2006) suggested that this was also the case for this species. For this reason, the gus $A$ fusion was constructed using the upstream region from vis $N$. For stabilized fusions, the promoter region of the gene was cloned into the parstabilized gusA fusion vector pFAJ1701 (Dombrecht et al., 2001) or pFus1par (Yost et al., 2004). Genome-integrated fusions were constructed by inserting a promoterless gusA gene within the corresponding flagellar gene (downstream of the promoter region) in a PJQ200SK gene construct. The gene fusion was integrated into the genome by homologous recombination. Correct integration was confirmed by Southern hybridization. The details of the generation of gene fragments used for constructing gusA-gene fusions are shown in Supplementary Table S2.

For the gusA assays involving the fusion strains in the wild-type and mutant backgrounds, the strains were grown in TY broth for $48 \mathrm{~h}$ at $30{ }^{\circ} \mathrm{C}$. The kinetics study was performed by growing the strains in TY broth at $30{ }^{\circ} \mathrm{C}$ with shaking (75 r.p.m.) and then samples were taken periodically for the gusA assay. $\beta$-Glucuronidase activity was measured as described by Jefferson et al. (1986) and modified by Yost et al. (2004). All data given are the means of at least triplicate experiments.

Nodulation conditions and gusA reporter gene assay for bacteroids. Plant experiments and gusA-gene expression assays for bacteroids were conducted as described by Yost et al. (2004). The nodules of plants inoculated with the R. leguminosarum gusA fusion strains were crushed and the supernatant containing the bacteroids was used for gusA assay. All data given in the tables and figures are means of triplicates, and nearly all experiments were repeated independently several times with similar results. The nodulation experiments were repeated at least twice, with similar trends.

\section{RESULTS AND DISCUSSION}

\section{Motility genes of $\boldsymbol{R}$. leguminosarum $\mathbf{3 8 4 1}$}

Analysis of the genome of $R$. leguminosarum 3841 (Young et al., 2006) showed that this strain has a large flagellar gene cluster (Fig. 1) similar to that of S. meliloti. This gene cluster in R. leguminosarum contains the majority of the genes involved in flagellar assembly and function, including four flagellin genes, the regulatory genes vis $N$, visR and rem, and the motility genes motABCfliK. Although $R$. leguminosarum and S. meliloti are closely related, they exhibit significant differences, such as the number of flagellin subunits and chemoreceptor genes. In addition to the four fla genes of $R$. leguminosarum (flaA to flaD) shown in Fig. 1, we have cloned three fla genes, flaE (pRL110518), flaH (RL3268) and flaG (RL 4729). Both flaH and flaG are chromosomally located in loci separate from the main flagellar regulon shown in Fig. 1. The other flagellin gene, flaE, is located on plasmid pRL11JI. The flagellin genes of $R$. leguminosarum encode proteins of around 300 amino acids, which are considerably shorter than the flagellins (321-401 amino acids) of S. meliloti (Capela et al., 2001). Five of the flagellin genes (flaABCEG) encode highly similar proteins, while $f l a D$ and $f l a H$ are quite divergent. There are also two complete che operons in R. leguminosarum (Miller et al., 2007) and 26 chemoreceptor genes, as opposed to nine in $S$. meliloti. We have completed
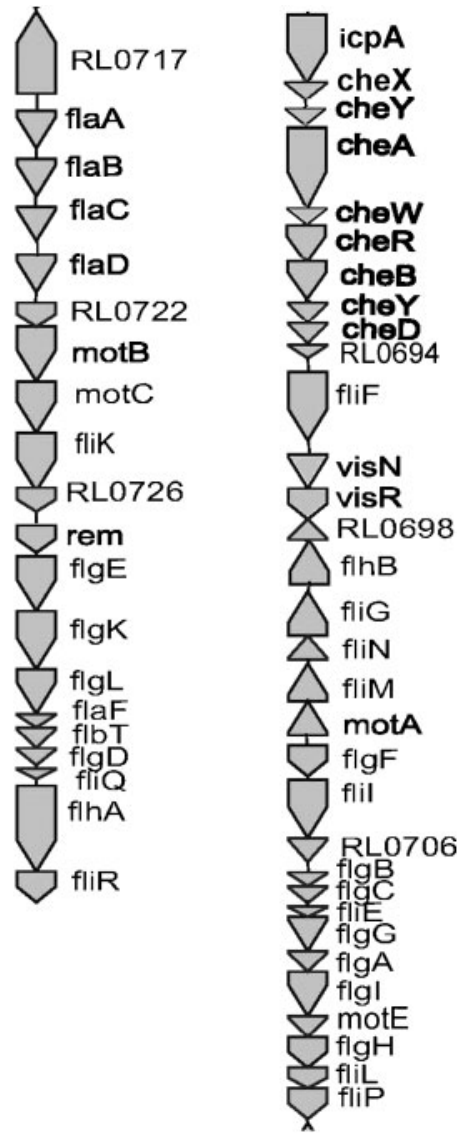

Fig. 1. Chromosomal region of $R$. leguminosarum 3841 containing the major chemotaxis, flagellin, motility and regulatory genes. Genes used in this study are in bold type. Adapted from RhizoDB (http://xbase.bham.ac.uk/rhizodb/).

sequence analyses and functional studies of the seven $\mathrm{fla}$ genes, which we describe in a separate paper (D. D. Tambalo and others, unpublished results).

\section{The roles of VisN, VisR and Rem in the regulation of the flagellar and motility genes of $R$. leguminosarum}

Very few studies have been done on the regulation of flagellar and chemotaxis genes in rhizobia, under both freeliving and symbiotic conditions. Three regulatory genes ( vis $N$, visR and rem) have been identified in S. meliloti, and these genes have been shown to function as global transcriptional activators of flagellar, motility and chemotaxis genes (Sourjik et al., 2000; Rotter et al., 2006). vis N and visR, the LuxR-type genes, encode proteins that control the expression of rem. The transcriptional activator Rem, which is expressed only during the exponential phase, acts as an intermediate between VisN/R and the motility and flagellar genes (Rotter et al., 2006). The roles of these regulators in the expression of flagellar and chemotaxis genes in R. leguminosarum were determined by cloning the 
orthologous genes from the sequenced strain 3841 and our laboratory strain VF39SM. We used both strains because these strains exhibit differences in the pattern of flagellation (Tambalo et al., 2010) and also in swarming motility (Tambalo et al., 2010). Strain VF39SM is peritrichously flagellated, while 3841 has one to two subpolar flagella.

BLAST searches showed that VisN (RL0696), VisR (RL0697) and Rem (RL0727) have 77, 71 and $92 \%$ similarity, respectively, to the corresponding proteins in $S$. meliloti. We constructed strains with mutations in the genes encoding the three transcriptional activators VisN, VisR and Rem for both 3841 and VF39SM. The VF39SM mutants were non-motile on swimming motility plates (Fig. 2) and appeared non-flagellated under TEM (Fig. 3), which indicated that these regulatory genes are necessary for flagellar synthesis. Complementation of the visN and visR mutants with cosmid pIJ9861 (Miller et al., 2007) resulted in restoration of motility.

We constructed transcriptional fusions for visN, rem, all flagellin genes, motA (RL0703) and $\operatorname{motB}$ (RL0723), and also used pre-existing fusions to chemotaxis genes $(m c p C$, mcpD, che1 and che2) (Yost et al., 2004; Miller et al., 2007) to determine whether $\mathrm{VisN} / \mathrm{R}$ and Rem control their transcription. The gusA-gene fusions were introduced into the strains carrying mutations in each of the regulatory genes and in wild-type backgrounds. The ratio of the promoter activity in the mutant to that in the wild-type background was obtained (Table 2). If the mutated gene did not exert transcriptional control over the promoter being tested, the ratio would be close to 1 , whereas the ratio would be less than 0.50 and close to 0 if the wild-type gene were needed for transcription. The gusA assays showed that for both VF39SM and 3841, the visN/visR promoter (visN and visR are in an operon) was negatively autoregulated, as indicated by higher promoter activities in the vis $N$ and visR mutant backgrounds (Table 2). We also confirmed that for both strains the expression of visN was not heavily influenced by Rem, as the values in rem mutant backgrounds, although significantly different statistically, were not radically lower than in the wild-type backgrounds. The expression of the 3841 and VF39SM rem promoters was lower in vis $N$ and visR mutant backgrounds, suggesting that rem is under the regulation of $\mathrm{VisN} / \mathrm{R}$, as is the case in $S$. meliloti. The expression levels of the four flagellin genes ( flaA, flaB, flaC and flaD) in both VF39SM and 3841 were reduced in the regulatory mutant backgrounds compared with gene expression in the wild-type backgrounds. For VF39SM, flaE and flaH did not exhibit a significant decrease in gene expression when the respective fusions were introduced into visN, visR and rem mutant backgrounds. flaG expression, while lower at a statistically significant level in the regulatory mutants, did not exhibit the same magnitude of dependence on VisN/R and Rem as that of other flagellin genes. For 3841, flaH was regulated by VisN/R and Rem, while flaE and flaG were not. Additionally, for both 3841 and VF39SM, motA and motB exhibited a reduction in gene expression in mutant backgrounds. The promoter activities of the $m c p C$ and che2 operon for both strains did not exhibit a significant decrease in gene expression in the mutant backgrounds, while those of the $m c p D$ and che1 operon were reduced.

These results obtained from the transcriptional studies revealed a hierarchy in the regulation of motility-related genes in R. leguminosarum (Fig. 4). The transcriptional activator VisN is on top of the hierarchy and exhibits autoregulation. VisN and VisR, which are believed to function as a heterodimer (Sourjik et al., 2000), appear to regulate the expression of rem. The flagellar genes of $R$.
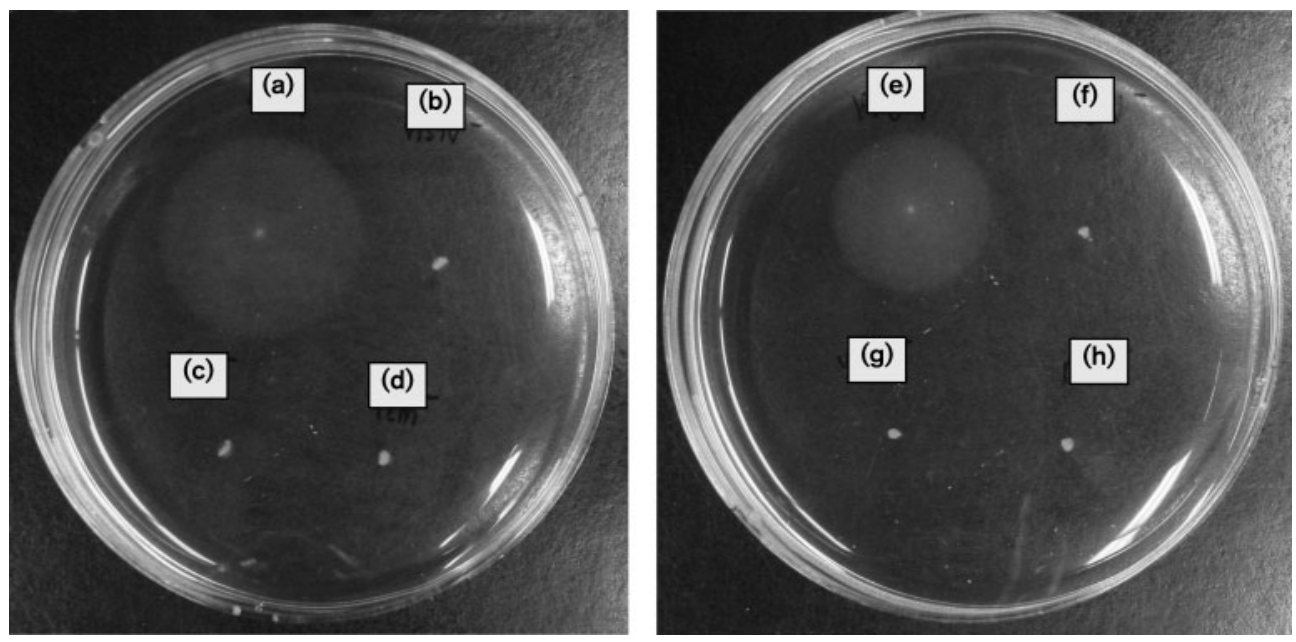

Fig. 2. Swimming motility plates for wild-type and regulatory mutants. (a) 3841 wild-type, (b) 3841 vis $N$ mutant, (c) 3841 vis $R$ mutant, (d) 3841 rem mutant, (e) VF39SM wild-type, (f) VF39SM visN mutant, (g) VF39SM visR mutant, (h) VF39SM rem mutant. 

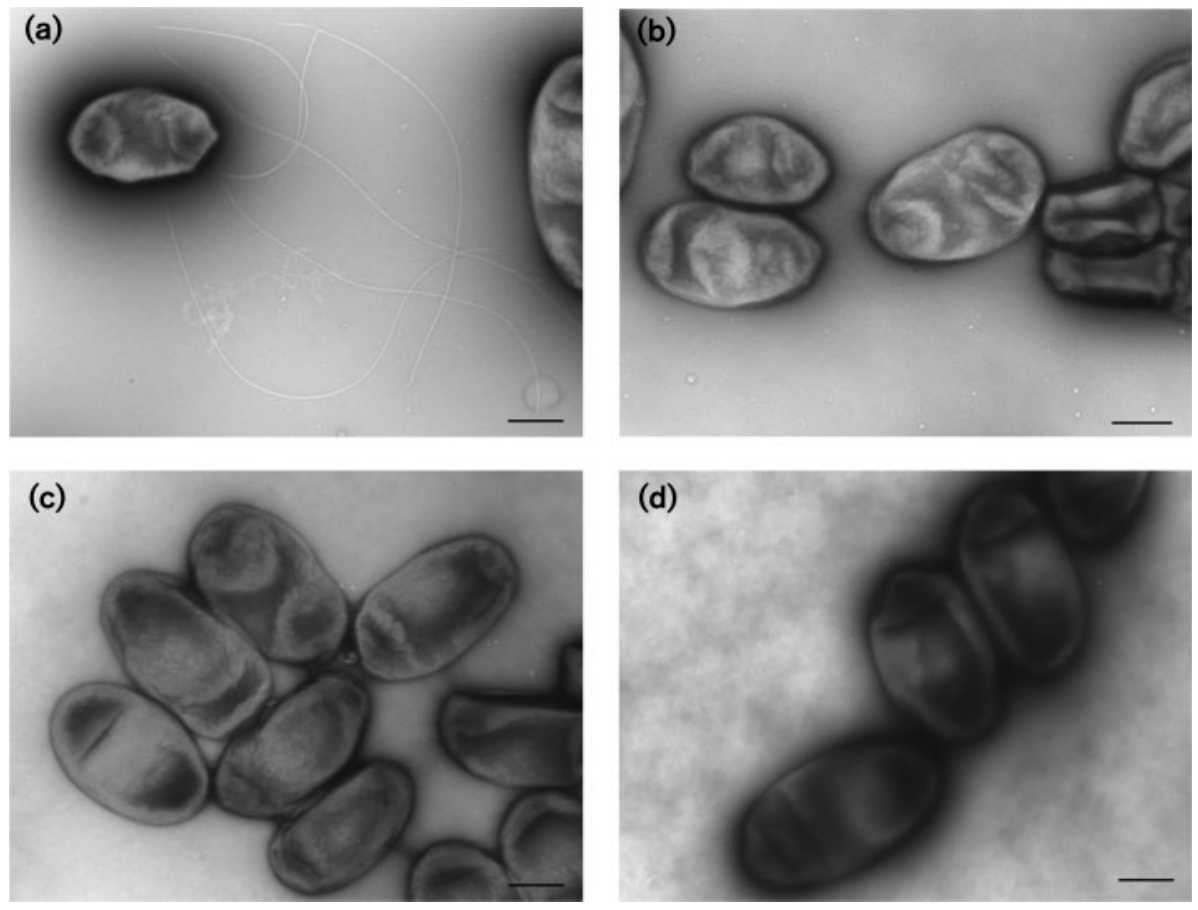

Fig. 3. TEM images of (a) R. leguminosarum VF39SM wild-type, (b) VF39SM visN mutant, (c) VF39SM visR mutant and (d) VF39SM rem mutant. Bars, $500 \mathrm{~nm}$.

leguminosarum are grouped into two categories: genes that are regulated by the VisN/R-Rem regulatory cascade and genes that are independent of this central motility regulation system. The genes that are positively controlled by VisN/R-Rem include flaA, flaB, flaC, flaD, motA, motB, $m c p D$ and che1, while che2, flaE, flaH (for VF39SM), flaG and $m c p C$ did not show clear evidence of transcriptional activation by these three regulatory genes. The four flagellin genes flaA, flaB, flaC and flaD, and motA, motB and che1, are located in the main flagellar motility region of strain 3841. The second che operon, flaH and flaG are located outside the main flagellar gene cluster, while both $f l a E$ and $m c p C$ are plasmid-borne. Hoang et al. (2008) have also reported a subset of flagellar genes ( $f l a F, f l b T$ and $f l a C$ ) that are not regulated by Rem in S. meliloti strain Rm8530. However, unlike the flagellar genes of $R$. leguminosarum, which exhibit complete independence from VisN/R-Rem regulation, the flagellar genes of $S$. meliloti that are not controlled by Rem are still under the regulation of VisN. It is therefore possible that in R. leguminosarum, there is a second regulatory system that controls the expression of the genes that are not under the VisN/R-Rem regulatory cascade. The regulation pattern described above is the same for VF39SM and 3841, except for $\mathrm{flaH}$, which is regulated by VisN/R and Rem in 3841 but not in VF39SM, and flaE, which is not under the described regulation in 3841 but repressed by Rem in VF39SM. Pairwise alignment of the promoter regions of the flagellar genes of VF39SM to the corresponding promoter regions of 3841 revealed that these regions have high homology (98-100\% identity), supporting the same regulation patterns observed in the transcription experiments.

We also noted that the transcript of the flaE gene from VF39SM increased to about threefold in a rem mutant background. This indicates that, contrary to the activator role of Rem in the expression of the other flagellin genes (flaABCD), Rem appears to repress the expression of flaE, though this effect may not be direct. Rotter et al. (2006) have shown through sequence alignment that Rem is a member of the OmpR family of transcriptional regulators. This family of regulators is characterized by a regulatory domain at the $\mathrm{N}$ terminus and a DNA-binding domain with a 'winged helix-turn-helix' pattern at the $\mathrm{C}$ terminus (Martínez-Hackert \& Stock, 1997; Rotter et al., 2006). The possible dual roles of Rem as an activator and a repressor of flagellin genes in R. leguminosarum have a precedent in E. coli, where it has been reported that OmpR can act as an activator and a repressor of a gene encoding a major outermembrane protein (OmpF) (Rampersaud et al., 1994). At low levels of phosphorylated OmpR, OmpR binds to the activator site of $o m p F$ to activate its expression. On the other hand, elevated levels of OmpR-phosphate would cause OmpR to bind to the repressor site, leading to $o m p F$ repression. A more recent study has also described opposite roles of OmpR in regulating the genes encoding outermembrane channels in Dickeya dadantii (formerly Erwinia chrysanthemi; Condemine \& Ghazi, 2007). While OmpR activates $k d g N$, which encodes an outer-membrane protein, it also acts as a repressor of $k d g M$, which encodes another 
Table 2. Expression of gus $A$ fusions to regulatory, flagellar, motility and chemotaxis genes in wild-type and mutant (vis $N$, vis $R$, rem) backgrounds

All values for wild-type strains are expressed as MU and are the means of at least three independent cultures ( \pm SD for the wild-type backgrounds). Expression levels in the visN, visR and rem mutants are shown as the ratio of mutant expression to that of the wild-type. Fusions were made using pFus1 unless otherwise indicated. The promoter regions were obtained from both VF39SM and 3841 unless otherwise indicated. ND, Not determined.

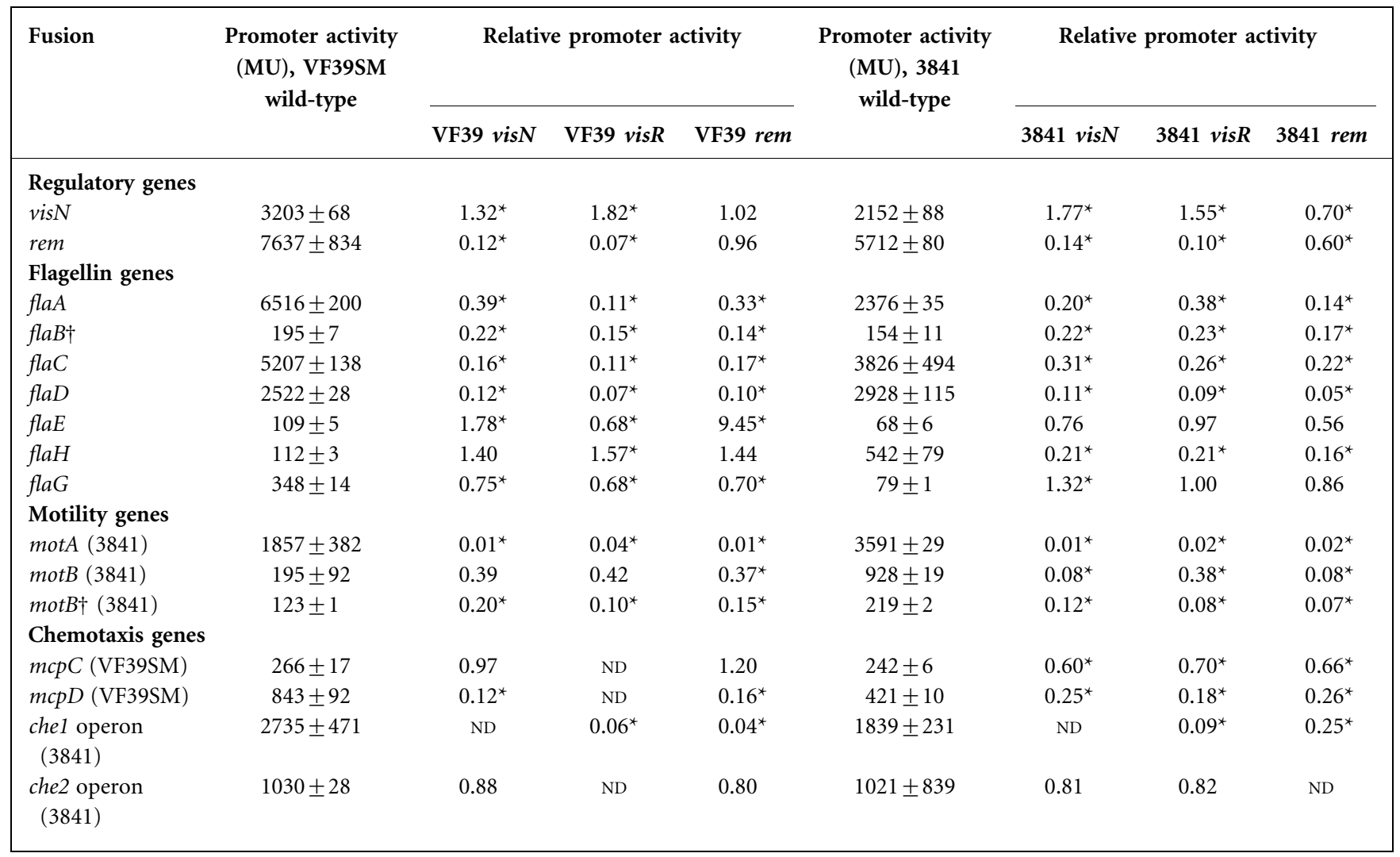

${ }^{\star}$ Difference between the wild-type and mutant is statistically significant at $P<0.001$ (Student's $t$ test).

$\dagger$ Fusion in pFAJ1701.

outer-membrane protein. Although the studies described above have demonstrated that OmpR can function as both an activator and a repressor for outer-membrane proteins, this is believed to be the first report of a dual function for an OmpR-type protein, specifically Rem, in the regulation of flagellin genes. It is curious that flaE expression does not increase in a vis $N$ or visR mutant, since Rem abundance should be low in such a mutant. However, this could be explained if VisN/R were required, either directly or indirectly, for expression of the flaE gene itself.

\section{Kinetics of flagellin and motility gene expression}

We looked at the expression patterns of flagellar genes, which represent the different classes in the regulatory scheme established in S. meliloti (Sourjik et al., 2000; Rotter et al., 2006). The regulatory genes vis $N$ and rem represent class I genes, whereas $m o t B$ and $f l a A$ represent class II and class III, respectively. $R$. leguminosarum strains carrying transcriptional fusions for flaA (pFus1::flaA) and $\operatorname{mot} B$
(pFAJ1701::motB) were constructed. The strains were grown in TY broth and their expression was measured at different growth phases using a gusA assay. The expression of flaA increased during the exponential phase and peaked at an enzyme activity of $6112 \pm 955$ Miller units (MU; Miller, 1972) (Fig. 5a). Promoter activity was still maintained at relatively high values $(66-88 \%)$ during the stationary phase. The same trend was observed for the expression of motB (Fig. 5b). The activity of the motB promoter increased during the exponential phase and the highest gene expression was observed after $48 \mathrm{~h}$ of incubation. The activity was also maintained at relatively high rates during the stationary phase before it dropped to around $60 \%$ at $288 \mathrm{~h}$.

The strains used in the above assay carried the gusA gene fusions in plasmids pFus1 and pFAJ1701. In these strains the genes are present in more than one copy; thus, the values for the promoter activities could have been higher than the actual expression values. To circumvent this 


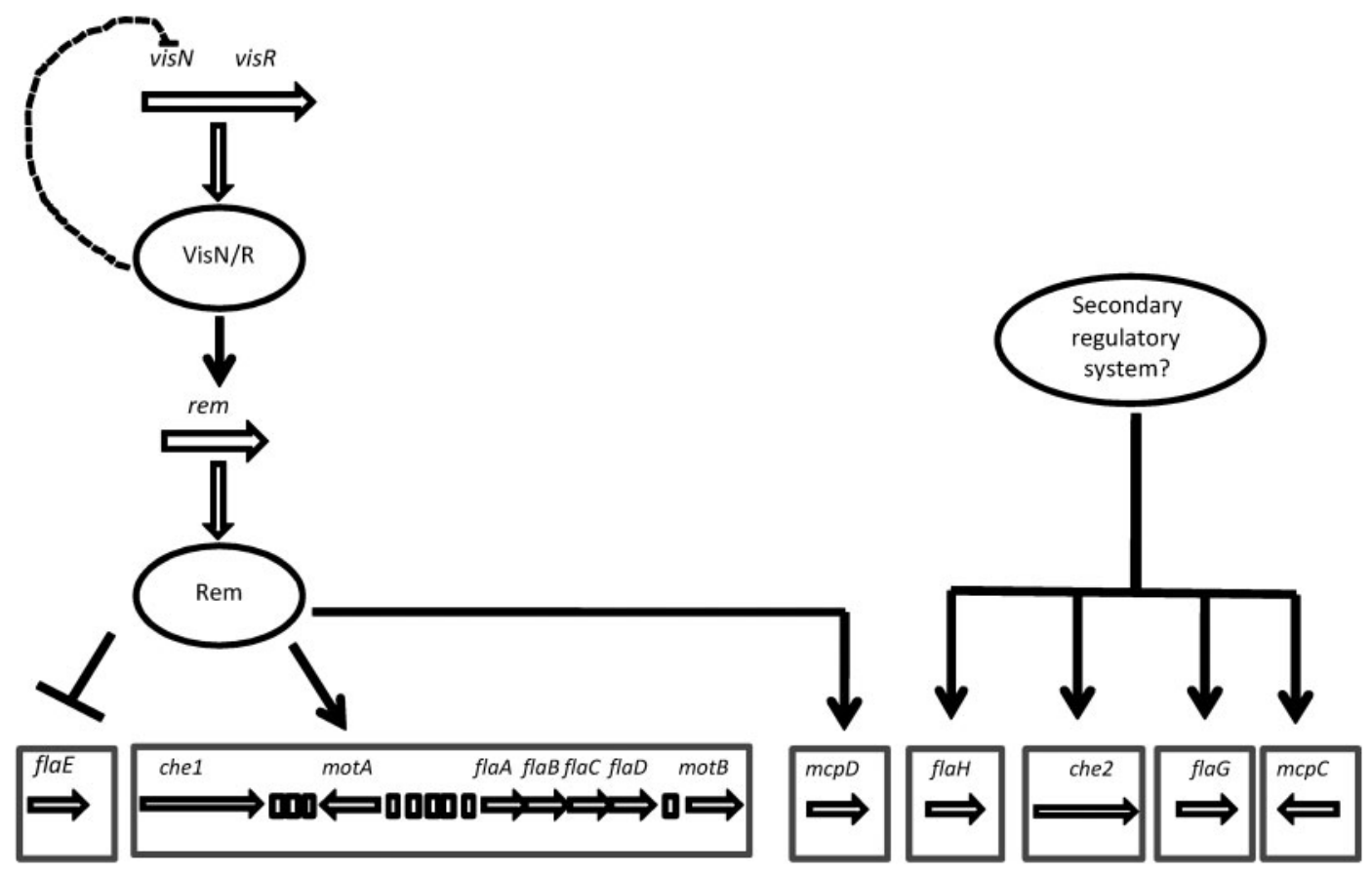

Fig. 4. Proposed model for the regulation of motility-related genes in R. leguminosarum. Genes are indicated as horizontal arrows and the corresponding gene products as ellipsoids. Translation to gene products is indicated by open vertical arrows. Black vertical arrows and bars indicate positive and negative regulation, respectively. Negative autoregulation of vis $N$ is shown as dotted arrows. All genes exhibited the same regulation pattern in 3841 and in VF39SM, except flaE and flaH. flaE is not regulated by VisN/R-Rem in 3841 but is repressed by Rem in VF39SM. flaH is under VisN/R-Rem regulation in 3841 but not in VF39SM.

problem, we repeated the assay for the flaA gene using strain A3SG/3841, which contains a genome-integrated flaA-gusA fusion. The highest promoter activity observed using the genome-integrated fusion was $935 \pm 11 \mathrm{MU}$, which was almost seven times lower than the highest value $(6112 \pm 955 \mathrm{MU})$ obtained using the flaA-gusA fusion in pFus1. Although the values differ in plasmid-borne and genome-integrated fusions, the same trend in gene expression was observed. Fig. 5(c) shows the kinetics of flaA gene expression until $201 \mathrm{~h}$ of incubation. The promoter activity started to increase at the onset of the exponential phase and reached its peak $33 \mathrm{~h}$ after incubation (late-exponential phase). During the stationary phase, gene expression of the chromosomally integrated flaA-gusA fusion in 3841 ranged from 61 to $88 \%$ of the highest value.

The transcriptional results described above indicate that the flagellin and motility genes are expressed throughout growth. During the lag and early exponential phases, flagellin and motility gene expression was minimal. At these stages of growth, the medium still contains a high concentration of nutrients and low concentrations of toxic metabolites, and therefore is still favourable for growth and reproduction (Amsler et al., 1993). The flagellin gene flaA and the motility gene $\operatorname{mot} B$ were continuously expressed during the stationary phase of the cultures. Wei \& Bauer (1998) have suggested the presence of compounds in stationary phase cultures that act as active inducers of motility. Thus, when filtrates from stationary phase cultures are added to $S$. meliloti cells under starvation conditions, cell motility is restored. They also indicated that the active substances in the culture filtrates are not necessarily metabolizable and do not need to be present at a certain concentration to prevent downregulation of motility under starvation conditions.

Since we had shown that $f l a A$ and $m o t B$ are controlled by the regulatory genes vis $N$, visR and rem, we determined whether these regulatory genes exhibit the same expression patterns as those of flaA and motB. The activities of both rem and vis $N$ promoters started to increase at the onset of the exponential phase and peaked at the onset of the stationary phase (Fig. 5d, e). The activity started to decrease during the stationary phase and then dropped to $33 \%$ of the highest promoter activity for both regulatory genes. VisN acts as a transcriptional activator of rem; hence, we observed a drop in the transcription of rem when the amount of vis $\mathrm{N}$ transcript decreased during the stationary phase. The expression patterns of vis $\mathrm{N}$ and rem differ from what has been described above for flaA and motB. The values obtained for vis $N$ and rem dropped 
(a)

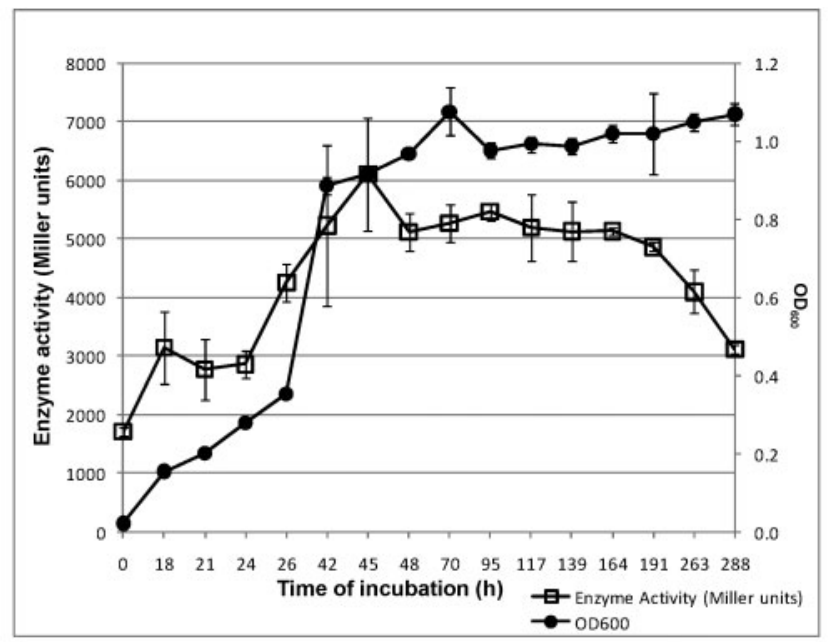

(b)

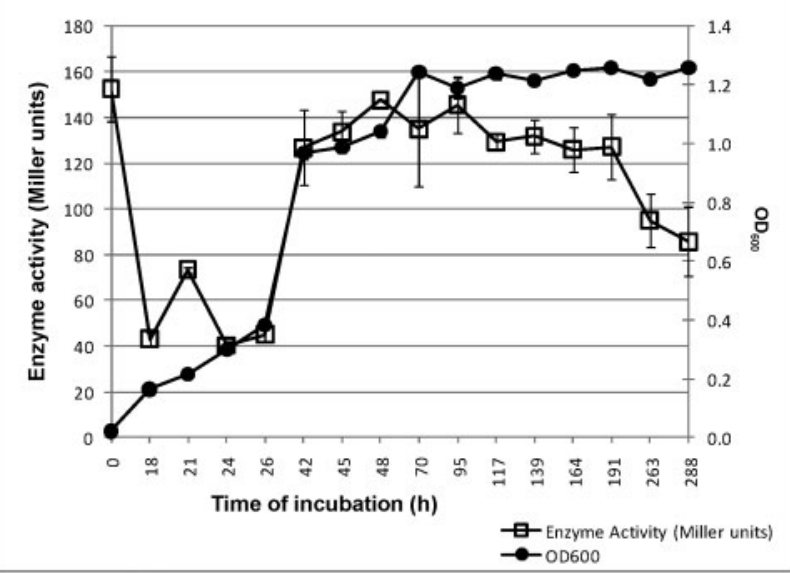

(e)

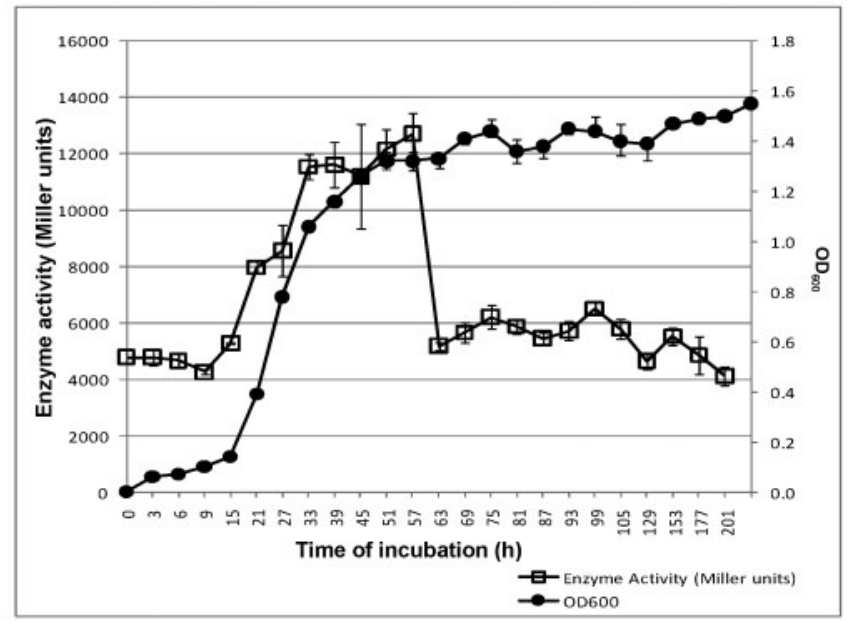

(c)

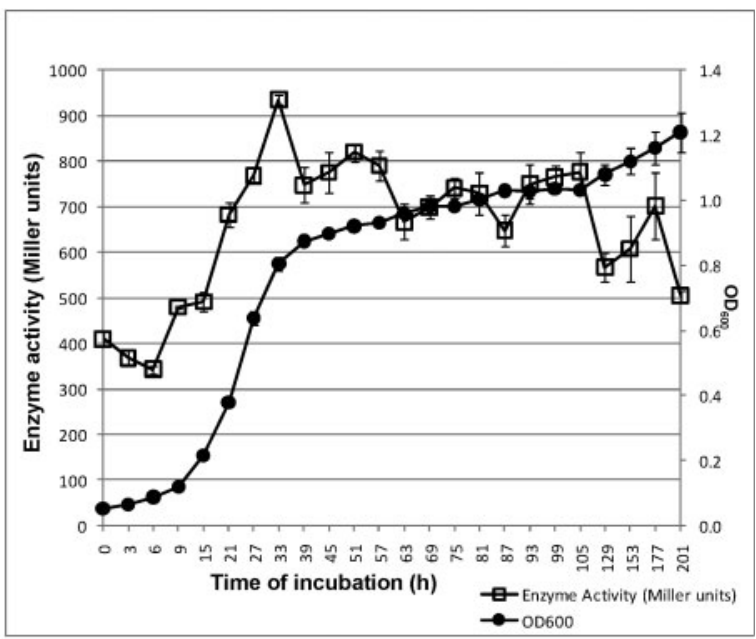

(d)

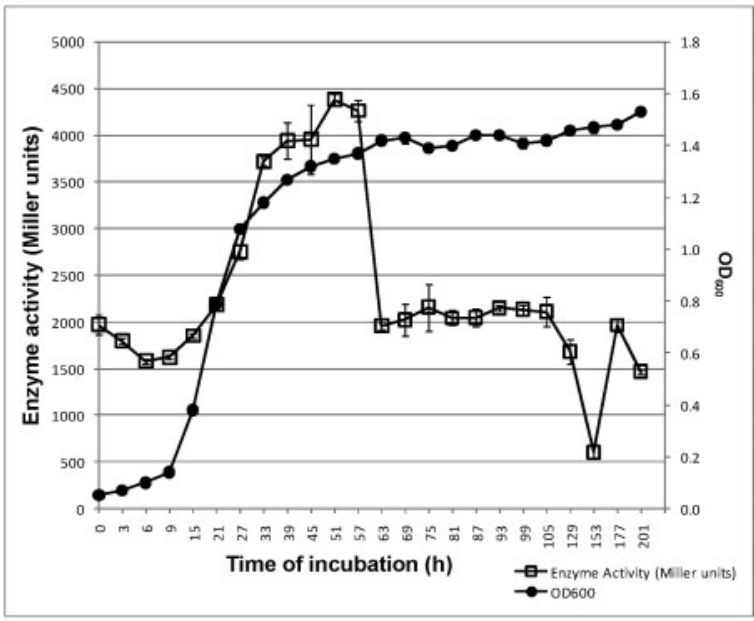

Fig. 5. Kinetics of flagellar gene expression in wild-type strains. Gene expression was measured as $\beta$-glucuronidase activity and expressed in MU. Values are means of three independent replicates; error bars, SD. (a) fla $A-$ gus $A$ fusion in VF39SM. The fla $A-$ gus $A$ gene fusion was constructed using pFus1 as vector. (b) $\operatorname{mot} B-$ gus $A$ fusion in VF39SM. The $\operatorname{mot} B-$ gus $A$ gene fusion was constructed using pFAJ1701. (c) The flaA promoter-gus $A$ gene fusion integrated into the genome of strain 3841. (d) vis $N$-gus $A$ fusion in 3841 . The vis $N$-gus $A$ gene fusion was constructed using pFus1. (e) rem-gus $A$ fusion in 3841 . The rem-gus $A$ gene fusion was constructed using pFus1. 
during the stationary phase, while flaA and motB exhibited relatively high expression at this phase. Although the transcription values for vis $\mathrm{N}$ and rem were lower in the stationary phase compared with expression during the exponential phase, we speculate that the amount of vis $\mathrm{N}$ and rem transcripts available might still be enough to drive the expression of the flagellin and motility genes.

To confirm the motility of the cells at the different growth phases, the cells were observed under a phase-contrast microscope. At the stationary phase, $30-40 \%$ of the cells exhibited swimming motility, which is approximately the same number as during exponential phase. TEM also revealed that the cells at the stationary phase were flagellated (Fig. 6). Most of the cells observed had two flagella per cell, which is less than the number (four to seven flagella per cell) observed for plate cultures. The broth culture was grown with shaking, thus some of the flagella could have been sheared off from the cells; the same lower number of flagella in broth culture was observed during exponential phase. There were also polysaccharides produced by the cells and salts in the TY medium that may have reacted with the stain used; thus, some flagella may not have been observed by TEM.

Similar growth-independent gene expression has been reported for $m c p$ fusions in $R$. leguminosarum (Yost et al., 2004). Motility alone is not sufficient for adaptation. The cells should be able to detect unfavourable changes to be able to respond; thus, at least some of the mcp genes of $R$. leguminosarum are also continuously expressed. Continuous cell motility has also been reported in E. coli. An increase in motility and flagellar expression has been observed in E. coli from the late-exponential phase to the early stationary phase (Amsler et al., 1993). Rhodobacter capsulatus, which is also a member of the $\alpha$-proteobacteria, exhibits the same gene expression pattern as $R$. leguminosarum. The transcripts for $f l g E$, which encodes the major hook protein, and for motB are higher in the lateexponential and stationary phases. The flagellin gene is also expressed in both the exponential and stationary phases. It has been suggested that the flagellin gene of Rhodobacter capsulatus is continuously transcribed because the flagellin protein is required in high amounts. In this way, a continuous pool of the protein is available for flagellum synthesis when the other flagellar proteins are transcribed (Lang \& Beatty, 2002).

The continuous flagellar gene expression described above is different from that reported in S. meliloti, where cell motility is confined within a window during the exponential phase (Sourjik et al., 2000; Rotter et al., 2006). Although both vis $N$ and visR are constitutively expressed throughout the entire growth of S. meliloti, other flagellar and motility genes (including rem) are not expressed in stationary or low-energy phases. Transcriptional analysis of rem in S. meliloti shows that its promoter activity increases rapidly at the start of the exponential phase and then abruptly drops by $75 \%$ before it decreases to a final activity below $10 \%$ (Rotter et al., 2006). Hoang et al. (2008) have also reported that the motility of $S$. meliloti is under the regulation of the ExpR/Sin quorum-sensing system. The expression of the motility genes is suppressed at high population densities (at late-exponential phase) by quorum sensing. On the other hand, expression of flagellin and motility genes in $R$. leguminosarum is still relatively high even at high population densities. This difference in the temporal expression of flagellar genes between $S$. meliloti and $R$. leguminosarum suggests that although these nodule

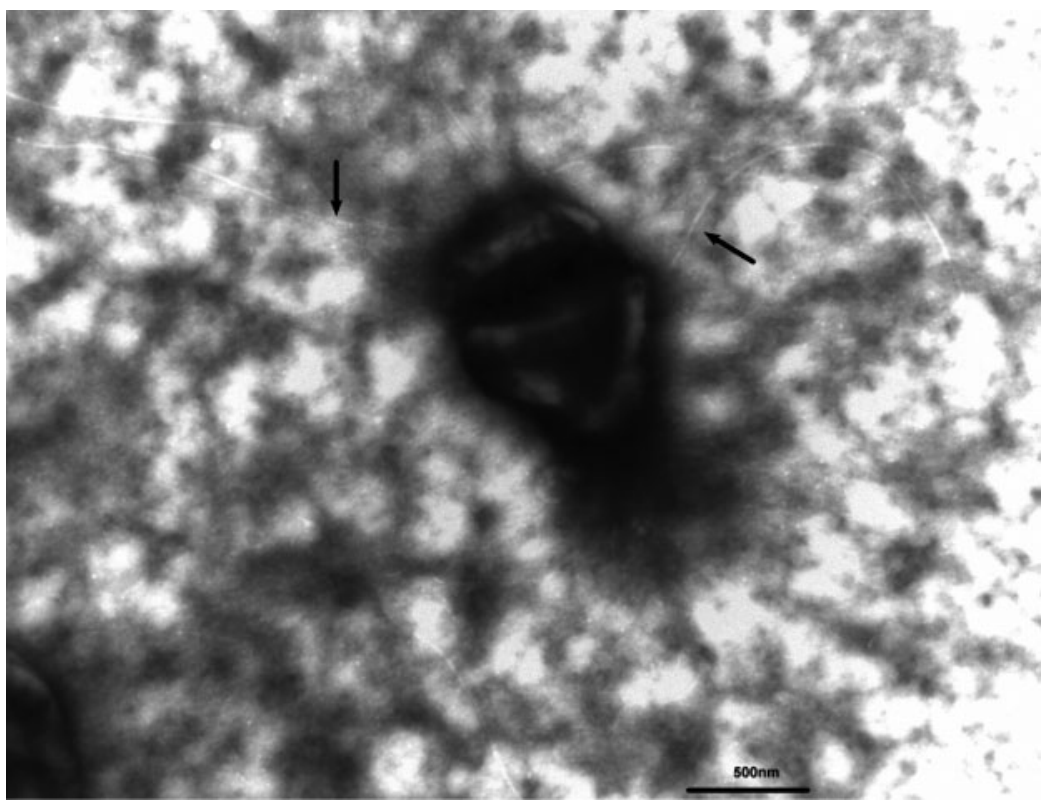

Fig. 6. TEM image of VF39SM at the stationary phase. Arrows show flagella. Bar, $500 \mathrm{~nm}$. 
bacteria are related, they employ different strategies when confronted with less favourable growth conditions such as those associated with the stationary phase. When the nutrients in the environment become scarce, motile bacteria need to choose between searching for new nutrient supplies and turning off motility to conserve energy. Chemotaxis and motility are energetically costly, requiring approximately 50 gene products (Wei \& Bauer, 1998) and approximately $2 \%$ of the cell's energy (Soutourina \& Bertin, 2003) for flagellar synthesis and function; hence the repression of motility in S. meliloti. On the other hand, continued chemotaxis and motility under free-living conditions, as in the case of $R$. leguminosarum, may result in finding more nutrients and a more favourable environment.

\section{Downregulation of regulatory genes under symbiotic conditions}

Since $R$. leguminosarum can live either freely or in symbiosis with its host plant, we also looked at the expression of motility genes during symbiosis. Our nodulation experiments showed that all of the motilityrelated genes studied were significantly repressed inside the nodules (Table 3). The promoter activities of flaA, flaB, flaC and mot $B$ were reduced 19-, eight-, 43- and eight-fold, respectively. The activity of the vis $N$ promoter exhibited as much as a 40-fold reduction for VF39SM. The reduction in the activity observed for 3841 was slightly lower, i.e. a $17-$ fold reduction. This could be attributed to the difference in the copy numbers of the gusA fusion vectors used for strains 3841 (pFAJ1701) and VF39SM (pFus1). We obtained a higher promoter activity under free-living conditions using pFus1 (data not shown). For rem, the expression was substantially repressed in the bacteroid, exhibiting as much as 40- to 63-fold reductions in 3841 and VF39SM, respectively. The downregulation of vis $\mathrm{N}$ and rem reported in this paper suggests that the symbiotic downregulation of motility-related genes such as the $m c p$ genes (Yost et al., 2004) as well as the fla and mot genes is mediated by repressing the expression of the genes that encode the global activators VisN/R and Rem.

\section{Conclusions}

The results from this study demonstrate a hierarchy in the regulation of motility-related genes in $R$. leguminosarum. The transcriptional activators VisN and VisR regulate the expression of rem. Rem, on the other hand, positively controls the expression of genes located within the main flagellar regulon, which includes flaA, flaB, flaC, flaD, motB and che1. Other genes, which are located outside of the main flagellar gene cluster in the chromosome (che2, flaH for VF39SM, and $f l a G)$ or are plasmid-borne $(m c p C)$, are not part of this regulatory cascade, and could be regulated by a secondary regulatory system. In addition, all genes exhibited the same regulation pattern in 3841 and in VF39SM, except flaE and flaH. flaE is not regulated by VisN/R-Rem in 3841 but its expression is repressed, though not necessarily directly, by Rem in VF39SM. flaH is under VisN/R-Rem regulation in 3841 but not in VF39SM. The kinetics experiment revealed that the flagellar genes (flaA, $m o t B, v i s N$ and rem) are expressed at all growth phases, indicating that $R$. leguminosarum is continuously motile under free-living conditions. In contrast, the regulatory genes $v i s \mathrm{~N}$ and $\mathrm{rem}$ are significantly downregulated when motility is not needed inside the nodules of the host plant.

\section{ACKNOWLEDGEMENTS}

We gratefully acknowledge the support for this work from a Natural Sciences and Engineering Research Council (NSERC) Discovery Grant to M. F. H. D. D. T. was supported by a Government of Canada graduate scholarship and the Bettina Bahlsen scholarship, and the work of A. E. S. and P. R. G. was supported by NSERC Undergraduate Student Research Awards (USRA). We thank Allan Downie, John Innes Centre, Norwich, UK, for the generous donation of pIJ9861, as well as Wei-Xiang Dong for his help with electron microscopy work and $\mathrm{Li}$ Han for her involvement in constructing the $\operatorname{mot} A$ fusion strains.

Table 3. Downregulation of flagellar and motility gene fusions in bacteroids

All values are expressed as MU and are the mean \pm SD of three independent replicates.

\begin{tabular}{|lcc|}
\hline Fusion & \multicolumn{2}{c|}{$\boldsymbol{\beta}$-Glucuronidase activity (MU) } \\
\cline { 2 - 3 } & Free-living & Bacteroids \\
\hline flaA-gusA fusion (3841); genome-integrated fusion & $935 \pm 11$ & $50 \pm 3$ \\
pFAJ1701::flaB (3841) & $155 \pm 11$ & $19 \pm 2$ \\
pFus1 ::flaC (3841) & $3826 \pm 494$ & $89 \pm 31$ \\
pFAJ1701:: motB (3841) & $217 \pm 5$ & $28 \pm 7$ \\
pFus1 :: visN promoter (VF39SM) & $3203 \pm 68$ & $109 \pm 55$ \\
pFAJ1701:: visN promoter (3841) & $852 \pm 33$ & $50 \pm 5$ \\
pFus1 :: rem promoter (VF39SM) & $7637 \pm 834$ & $169 \pm 94$ \\
pFus1 :: rem promoter (3841) & $6962 \pm 133$ & $276 \pm 110$ \\
\hline
\end{tabular}




\section{REFERENCES}

Ames, P. \& Bergman, K. (1981). Competitive advantage provided by bacterial motility in the formation of nodules by Rhizobium meliloti. J Bacteriol 148, 728-729.

Ames, P., Schluederberg, S. A. \& Bergman, K. (1980). Behavioral mutants of Rhizobium meliloti. J Bacteriol 141, 722-727.

Amsler, C. D., Cho, M. \& Matsumura, P. (1993). Multiple factors underlying the maximum motility of Escherichia coli as cultures enter post-exponential growth. J Bacteriol 175, 6238-6244.

Bauer, W. D. \& Caetano-Anollés, G. (1990). Chemotaxis, induced gene expression, and competitiveness in the rhizosphere. Plant Soil 129, 45-52.

Berg, H. C. (2003). The rotary motor of bacterial flagella. Annu Rev Biochem 72, 19-54.

Beringer, J. E. (1974). R factor transfer in Rhizobium leguminosarum. J Gen Microbiol 84, 188-198.

Caetano-Anollés, G., Wall, L. G., Micheli, A. T. D., Macchi, E. M., Bauer, W. D. \& Favelukes, G. (1988). Role of motility and chemotaxis in efficiency of nodulation by Rhizobium meliloti. Plant Physiol 86, $1228-1235$.

Capela, D., Barloy-Hubler, F., Gouzy, J., Bothe, G., Ampe, F., Batut, J., Boistard, P., Becker, A., Boutry, M. \& other authors (2001). Analysis of the chromosome sequence of the legume symbiont Sinorhizobium meliloti strain 1021. Proc Natl Acad Sci U S A 98, 9877-9882.

Condemine, G. \& Ghazi, A. (2007). Differential regulation of two oligogalacturonate outer membrane channels, KdgN and KdgM, of Dickeya dadantii (Erwinia chrysanthemi). J Bacteriol 189, 5955-5962.

Deakin, W. J., Parker, V. E., Wright, E. L., Ashcroft, K. J., Loake, G. J. \& Shaw, C. H. (1999). Agrobacterium tumefaciens possesses a fourth flagellin gene located in a large gene cluster concerned with flagellar structure, assembly and motility. Microbiology 145, 1397-1407.

Dombrecht, B., Vanderleyden, J. \& Michiels, J. (2001). Stable RK2derived cloning vectors for the analysis of gene expression and gene function in Gram-negative bacteria. Mol Plant Microbe Interact 14, 426-430.

Easter, C. L., Sobecky, P. A. \& Helinski, D. R. (1997). Contribution of different segments of the par region to stable maintenance of the broad-host-range plasmid RK2. J Bacteriol 179, 6472-6479.

Eggenhofer, E., Rachel, R., Haslbeck, M. \& Scharf, B. (2006). MotD of Sinorhizobium and related $\alpha$-proteobacteria is the flagellar-hooklength regulator and therefore assigned as FliK. J Bacteriol 188, 21442153.

Fellay, R., Frey, J. \& Krisch, H. (1987). Interposon mutagenesis of soil and water bacteria: a family of DNA fragments designed for in vitro insertional mutagenesis of Gram-negative bacteria. Gene 52, 147-154.

Hoang, H. H., Gurich, N. \& González, J. E. (2008). Regulation of motility by the ExpR/Sin quorum-sensing system in Sinorhizobium meliloti. J Bacteriol 190, 861-871.

Jefferson, R. A., Burgess, S. M. \& Hirsh, D. (1986). Betaglucuronidase from Escherichia coli as a gene-fusion marker. Proc Natl Acad Sci U S A 83, 8447-8451.

Jones, J. D. \& Gutterson, N. (1987). An efficient mobilizable cosmid vector, pRK7813, and its use in a rapid method for marker exchange in Pseudomonas fluorescens strain HV37a. Gene 61, 299-306.

Kutsukake, K., Ohya, Y. \& lino, T. (1990). Transcriptional analysis of the flagellar regulon of Salmonella typhimurium. J Bacteriol 172, 741747.

Lang, A. S. \& Beatty, J. T. (2002). A bacterial signal transduction system controls genetic exchange and motility. J Bacteriol 184, 913918.
Liu, R., Tran, V. M. \& Schmidt, E. L. (1989). Nodulating competitiveness of a non-motile Tn7 mutant Bradyrhizobium japonicum in nonsterile soil. Appl Environ Microbiol 55, 1895-1900.

Macnab, R. M. (1992). Genetics and biogenesis of bacterial flagella. Annu Rev Genet 26, 131-158.

Malek, W. (1992). The role of motility in the efficiency of nodulation by Rhizobium meliloti. Arch Microbiol 158, 26-28.

Martínez-Hackert, E. \& Stock, A. M. (1997). Structural relationships in the OmpR family of winged-helix transcription factors. J Mol Biol 269, 301-312.

Mellor, H. Y., Glenn, A. R., Arwas, R. \& Dilworth, M. J. (1987). Symbiotic and competitive properties of Rhizobium trifolii. Arch Microbiol 148, 34-39.

Miller, J. H. (1972). Experiments in Molecular Genetics. Cold Spring Harbor, NY: Cold Spring Harbor Laboratory.

Miller, L. D., Yost, C. K., Hynes, M. F. \& Alexandre, G. (2007). The major chemotaxis gene cluster of Rhizobium leguminosarum bv. viciae is essential for competitive nodulation. Mol Microbiol 63, 348-362.

Morita, R. Y. (1988). Bioavailability of energy and its relationship to growth and starvation survival in nature. Can J Microbiol 34, 436-441.

Pleier, E. \& Schmitt, R. (1989). Identification and sequence analysis of two related flagellin genes in Rhizobium meliloti. J Bacteriol 171, 14671475.

Pleier, E. \& Schmitt, R. (1991). Expression of two Rhizobium meliloti flagellin genes and their contribution to the complex filament structure. J Bacteriol 173, 2077-2085.

Poole, P., Blyth, A., Reid, J. \& Walters, K. (1994). myo-Inositol catabolism and catabolite regulation in Rhizobium leguminosarum bv. viciae. Microbiology 140, 2787-2795.

Prentki, P. \& Krisch, H. M. (1984). In vitro insertional mutagenesis with a selectable DNA fragment. Gene 29, 303-313.

Priefer, U. B. (1989). Genes involved in lipopolysaccharide production and symbiosis are clustered on the chromosome of Rhizobium leguminosarum biovar viciae VF39. J Bacteriol 171, 6161-6168.

Pruss, B. M. \& Matsumura, P. (1997). Cell cycle regulation of flagellar genes. J Bacteriol 179, 5602-5604.

Quandt, J. \& Hynes, M. F. (1993). Versatile suicide vectors which allow direct selection for gene replacement in Gram-negative bacteria. Gene 127, 15-21.

Rampersaud, A., Harlocker, S. L. \& Inouye, M. (1994). The OmpR protein of Escherichia coli binds to sites in the ompF promoter region in a hierarchical manner determined by its degree of phosphorylation. J Biol Chem 269, 12559-12566.

Reeve, W. G., Tiwari, R. P., Worsley, P. S., Dilwoth, M. J., Glenn, A. R. \& Howieson, J. G. (1999). Constructs for insertional mutagenesis, transcriptional signal localization, and gene regulation studies in root nodule and other bacteria. Microbiology 145, 1307-1316.

Rotter, C., Muhlbacher, S., Salamon, D., Schmitt, R. \& Scharf, B. (2006). Rem, a new transcriptional activator of motility and chemotaxis in Sinorhizobium meliloti. J Bacteriol 188, 6932-6942.

Sambrook, J., Fritsch, E. F. \& Maniatis, T. (1989). Molecular Cloning, a Laboratory Manual, 2nd edn. Cold Spring Harbor, NY: Cold Spring Harbor Laboratory.

Scharf, B., Schuster-Wolff-Buhring, H., Rachel, R. \& Schmitt, R. (2001). Mutational analysis of the Rhizobium lupini H13-3 and Sinorhizobium meliloti flagellin genes: importance of flagellin A for flagellar filament structure and transcriptional regulation. J Bacteriol 183, 5334-5342.

Shi, W., Zhou, Y., Wild, J., Adler, J. \& Gross, C. A. (1992). DnaK, DnaJ, and GrpE are required for flagellum synthesis in Escherichia coli. Bacteriol 174, 6256-6263. 
Silverman, M. \& Simon, M. (1974). Characterization of Escherichia coli flagellar mutants that are insensitive to catabolite repression. J Bacteriol 120, 1196-1203.

Simon, R., Priefer, U. \& Puhler, A. (1983). A broad host range mobilization system for in vivo genetic engineering: transposon mutagenesis in Gram negative bacteria. Nat Biotechnol 1, 784-791.

Soby, S. \& Bergman, K. (1983). Motility and chemotaxis of Rhizobium meliloti in soil. Appl Environ Microbiol 46, 995-998.

Sourjik, V. \& Schmitt, R. (1996). Different roles of CheY1 and CheY2 in the chemotaxis of Rhizobium meliloti. Mol Microbiol 22, 427-436.

Sourjik, V., Sterr, W., Platzer, J., Bos, l., Haselbeck, M. \& Schmitt, R. (1998). Mapping of 41 chemotaxis, flagellar and motility genes to a single region of the Sinorhizobium meliloti chromosome. Gene 223 , 283-290.

Sourjik, V., Muschler, P., Scharf, B. \& Schmitt, R. (2000). VisN and VisR are global regulators of chemotaxis, flagellar, and motility genes in Sinorhizobium (Rhizobium) meliloti. J Bacteriol 182, 782-788.

Soutourina, O. A. \& Bertin, P. N. (2003). Regulation cascade of flagellar expression in Gram-negative bacteria. FEMS Microbiol Rev 27, 505-523.

Soutourina, O. A., Semenova, E. A., Parfenova, V. V., Danchin, A. \& Bertin, P. (2001). Control of bacterial motility by environmental factors in polarly flagellated peritrichous bacteria isolated from Lake Baikal. Appl Environ Microbiol 67, 3852-3859.

Tambalo, D. D., Yost, C. K. \& Hynes, M. F. (2010). Characterization of swarming motility in Rhizobium leguminosarum bv. viciae. FEMS
Microbiol Lett (in press)..http://dx.doi.org/10.1111/j.1574-6968.2010. 01982.x

Thorne, S. H. \& Williams, H. D. (1997). Adaptation to nutrient starvation in Rhizobium leguminosarum bv. phaseoli: analysis of survival, stress resistance, and changes in macromolecular synthesis during entry to and exit from stationary phase. J Bacteriol 179, 68946901.

Walker, T. S., Bais, H. P., Grotewold, E. \& Vivanco, J. M. (2003). Root exudation and rhizosphere biology. Plant Physiol 132, 44-51.

Wei, X. \& Bauer, W. D. (1998). Starvation-induced changes in motility, chemotaxis, and flagellation of Rhizobium meliloti. Appl Environ Microbiol 64, 1708-1714.

Yost, C. K., Rochepeau, P. \& Hynes, M. F. (1998). Rhizobium leguminosarum contains a group of genes that appear to code for methyl-accepting chemotaxis proteins. Microbiology 144, 19451956.

Yost, C. K., Del Bel, K. L., Quandt, J. \& Hynes, M. F. (2004). Rhizobium leguminosarum methyl-accepting chemotaxis protein genes are downregulated in the pea nodule. Arch Microbiol 182, 505-513.

Young, J. P., Crossman, L. C., Johnston, A. W., Thomson, N. R., Ghazoui, Z. F., Hull, K. H., Wexler, M., Curson, A. R., Todd, J. D. \& other authors (2006). The genome of Rhizobium leguminosarum has recognizable core and accessory components. Genome Biol 7, R34.

Edited by: H.-M. Fischer 